\title{
In vivo regulation of neutrophil apoptosis by C5a during sepsis
}

\author{
Ren-Feng Guo, ${ }^{1}$ Lei Sun, Hongwei Gao, Kevin X. Shi, Daniel Rittirsch, Vidya J. Sarma, \\ Firas S. Zetoune, and Peter A. Ward ${ }^{1}$
}

University of Michigan Medical School, Department of Pathology, Ann Arbor, Michigan, USA

\begin{abstract}
Delayed neutrophil apoptosis is characteristic of sepsis and may accentuate organ injury. It has been shown that PI-3K and MAPK pathways provide survival signaling in neutrophils. In this study, we demonstrate that neutrophils isolated from septic rats are resistant to apoptosis in comparison with the cells from normal animals. In contrast to normal serum, septic sera induced strong phosphorylation of AKT and p44/42 in neutrophils obtained from normal rats, resulting in marked resistance of these cells to apoptosis. Protection from apoptosis by septic sera was abrogated completely by inhibition of PI-3K and partially diminished by MEK inhibition. Increased neutrophil survival in septic rats was associated with increased levels of Bcl-xL in neutrophils and decreased levels of Bim expression. In vivo blockade of $\mathrm{C5a}$ in cecal ligation and puncture rats by anti-C5a antibody markedly restored the susceptibility of neutrophils to undergo apoptosis. C5a activated AKT and p44/42 and also enhanced $X$-linked inhibitor of apoptosis expression in neutrophils. LPS and C5a were able to induce Bcl-xL expression. Thus, neutrophil survival signals derived from effects of septic sera could be linked to activation of ERK1/2 and PI-3K, increased antiapoptotic protein expression, and ultimately, delayed neutrophil apoptosis. J. Leukoc. Biol. 80: 1575-1583; 2006.
\end{abstract}

Key Words: cell signaling $\cdot$ survival $\cdot$ phagocyte $\cdot$ inflammation

\section{INTRODUCTION}

Neutrophils are major effector cells at the frontier of the immune response and play a critical role in host defenses against invading microorganisms. Under inflammatory settings such as ischemia/reperfusion injury and acute respiratory distress syndrome, neutrophils tend to accumulate in inflamed tissues, where they exert two significant functions: eradication of invading microorganisms and induction of tissue damage. Tissue injury can be caused by proteases and reactive oxygen radicals released from activated neutrophils. In addition, neutrophils are able to produce cytokines and chemokines that affect the process of inflammation [1]. It is generally believed that neutrophil accumulation is a hallmark of the phlogistic process and plays an essential role in tissue injury.
Apoptosis is an important process for removal of neutrophils in inflamed tissues. This not only diminishes the release of inflammatory mediators and enzymes but also removes neutrophils from tissues [2]. Under normal conditions, nearly $1-2 \times$ $10^{11}$ human neutrophils are generated each day. Their lifespan is short (24-36 h) in the circulation [3]. However, in certain inflammatory states, neutrophils show a prolonged lifespan. Delayed apoptosis of neutrophils in the lung has been observed after hemorrhage or endotoxemia [4]. Neutrophil apoptosis in patients with systemic inflammatory response syndrome (SIRS) decreases significantly when compared with that in healthy controls, and the plasma from these patients can inhibit apoptosis of normal neutrophils, suggesting that survival signals are derived from inflammatory mediators in blood [5]. Many inflammatory cytokines and chemokines have been found to render neutrophils survival signals. For instance, TNF- $\alpha$, IL$1 \beta$, IL-6, IFN- $\gamma$, GM-CSF, G-CSF, and IL-8 have been shown to prolong the lifespan of circulating neutrophils [6]. Other inflammatory mediators such as leukotriene B4 (LTB4), LPS, and C5a can also delay neutrophil apoptosis $[6,7]$. Delayed neutrophil apoptosis may contribute to SIRS and organ damage, given the facts that antineutrophil treatment attenuates lung and liver damage [8] and improves survival in the setting of experimental sepsis [9].

C5a is a potent, inflammatory peptide with a broad spectrum of biological functions [10]. C5a induces neutrophil chemoattraction, an oxidative burst (consumption of $\mathrm{O}_{2}$ and production of $\mathrm{H}_{2} \mathrm{O}_{2}$ ), enhancement of phagocytosis, and release of granule enzymes. C5a is also a strong vasodilator. During sepsis, the C5a receptor $(\mathrm{C} 5 \mathrm{aR})$ has been found to be up-regulated in major organs (lungs, liver, kidneys, heart) [11], and C5aR expression on neutrophils is diminished greatly [12]. Recently, C5a has been found to suppress neutrophil apoptosis [13, 14]. However, all the studies were done in an in vitro setting. The mechanism by which C5a exerts its antiapoptotic effect on neutrophils in the setting of sepsis is largely unknown. The current study was designed to evaluate the role of C5a in neutrophil apoptosis during sepsis and to identify intracellular events responsible for delayed neutrophil apoptosis during septic conditions.

\footnotetext{
${ }^{1}$ Correspondence: Department of Pathology, The University of Michigan Medical School, 1301 Catherine Road, Ann Arbor, MI 48109-0602, USA. E-mail: grf@med.umich.edu or pward@umich.edu

Received January 27, 2006; revised July 10, 2006; accepted August 25, 2006 doi: 10.1189/jlb.0106065
} 


\section{MATERIALS AND METHODS}

\section{Reagents}

RPMI-1640 medium and FBS were obtained from Gibco Life Technologies (Rockville, MD). MEK1/2 inhibitor U0126 and PI-3K inhibitor LY294002 were purchased from Cell Signaling Technology Inc. (Beverly, MA). Antibodies to rat AKT, phospho-AKT, p44/42, phospho-p44/42, X-linked inhibitor of apoptosis (XIAP), and Smac/Diablo were obtained from Cell Signaling Technology Inc., and antibody to GAPDH was obtained from Abcam, Inc. (Cambridge, MA). Recombinant rat C5a was produced in our laboratory [15]. LPS was obtained from Sigma-Aldrich (St. Louis, MO; Escherichia coli 026:B6 cell culture-tested, purified by gel-filtration chromatography, $\gamma$-irradiated).

\section{Rat model of cecal ligation and puncture (CLP)}

Male Long-Evans-specific, pathogen-free rats (275-300 g, Harlan, Inc., Indianapolis, IN) were used in all studies. Anesthesia was induced by i.p. administration of ketamine $(20 \mathrm{mg} / 100 \mathrm{~g}$ body weight). After shaving the abdomen and application of a topical disinfectant, a 2 -cm midline incision was made, and the cecum was identified and ligated below the ileocecal valve, with care being taken not to occlude the bowel or its vascular supply. The cecum was then subjected to a single through-and-through perforation with an 18-gauge needle. After repositioning the bowel, the abdominal incision was closed in layers with a plain gut surgical suture 4-0 (Ethicon, Inc., Somerville, NJ) and metallic clips. Sham animals underwent the same procedure in the absence of CLP. Before and after surgery, animals had unlimited access to food and water. Animals receiving anti-C5a antibody treatment were injected i.v. at the time of the CLP procedure with $500 \mu \mathrm{g}$ rabbit anti-rat C5a IgG (against the carboxylterminal region; purified and characterized as described previously [16]) or with $500 \mu \mathrm{g}$ preimmune rabbit IgG.

\section{Isolation of rat blood neutrophils}

Whole blood from control rats or CLP rats was drawn into syringes containing the anticoagulant, acid citrate glucose (Baxter Health Care, Mundelein, IL). Neutrophils were isolated using Ficoll-Paque gradient centrifugation (Pharmacia Biotech, Uppsala, Sweden) and dextran sedimentation. After hypotonic lysis of residual RBC, the neutrophils were used for further studies. Cell purity $(>95 \%)$ and viability $(>98 \%)$ were evaluated by the Trypan blue exclusion method.

\section{Cell culture and stimulation}

The neutrophils were maintained in RPMI 1640 supplemented with 10\% FCS, $2 \mathrm{mM}$ L-glutamine, $100 \mathrm{U} / \mathrm{ml}$ penicillin, and $100 \mu \mathrm{g} / \mathrm{ml}$ streptomycin. A final concentration of $1 \times 10^{6} \mathrm{cells} / \mathrm{ml}$ was used for stimulation at $37^{\circ} \mathrm{C}$ in a humidified $\mathrm{CO}_{2}$ incubator $\left(5 \% \mathrm{CO}_{2}\right.$ and $95 \%$ air). As indicated, cells were incubated with $20 \mu \mathrm{M}$ MEK1/2 inhibitor (U0126) or $50 \mu \mathrm{M}$ PI-3K inhibitor (LY294002). These doses have been found to block MEK1/2 and PI-3K activation effectively in neutrophils $[17,18]$. For in vitro apoptosis assay, the neutrophils were incubated in an ultra-low attachment polystyrene plate (Corning Inc., Corning, NY) for $20 \mathrm{~h}$, and apoptosis assessment was subsequently performed using binding of Annexin V (Ax).

\section{Collection of serum samples from septic animals}

After induction of sepsis, animals were killed at the indicated time-points after CLP, and blood was drawn from the inferior caval vein. Blood samples were allowed to clot on ice for $4 \mathrm{~h}$ before centrifugation at $3000 \mathrm{rpm}$ for $15 \mathrm{~min}$ at $4^{\circ} \mathrm{C}$. Serum was collected and frozen immediately at $-80^{\circ} \mathrm{C}$ until use.

\section{Detection of neutrophil apoptosis by flow cytometry analysis}

Neutrophils $\left(1 \times 10^{6}\right)$ were stained with Ax conjugated to FITC and propidium iodide (PI) by using the Ax-FLUOS staining kit (Boehringer-Mannheim Corp., Germany), using the manufacturer's instructions. Immediately after staining, the cells were analyzed on a flow cytometer (Coulter Corp., Miami, FL) using $488 \mathrm{~nm}$ excitation and a 525-nm bandpass filter for FITC and a 620-nm filter for PI detection. Data were analyzed by using WinList computer software (Verity Software House Inc., Topsham, ME). Electronic compensation of the instrument was carried out to exclude overlapping of the two emission spectra. Cell debris was eliminated by gating according to side-scatter and forwardscatter detection. The regions for Ax-positive only, PI-positive only, doublepositive, and double-negative were created by single-parameter analysis with Ax-FITC or PI. Neutrophil apoptosis is presented by the percentage of Axpositive cells, which include early and late apoptotic cells.

\section{Western blot analysis}

Approximately $5 \times 10^{6}$ cells per condition were used for whole cell lysis using $100 \mu \mathrm{l}$ Laemmli buffer containing 5\% 2-ME. Lysates were separated on a NuPAGE 4-12\% bis-Tris gel (Invitrogen, Carlsbad, CA), and proteins were transferred to a polyvinylidene difluoride membrane. Nonspecific binding sites were blocked with TBST $(40 \mathrm{mM}$ Tris, $\mathrm{pH}$ 7.6, $300 \mathrm{mM} \mathrm{NaCl}$, and $0.1 \%$ Tween 20 ) containing $5 \%$ nonfat dry milk for $12 \mathrm{~h}$ at $4^{\circ} \mathrm{C}$. Membranes were incubated with the following antibodies in a 1:1000 or 1:2000 dilution: polyclonal rabbit anti-BAX (PharMingen, San Diego, CA), monoclonal mouse anti-Bcl-xL (PharMingen), polyclonal rabbit anti-Bad (Santa Cruz Biotechnology Inc., CA), polyclonal rabbit anti-Bim (PharMingen), and antibodies to AKT, phosphoAKT, p44/p42, phospho-p44/p42, XIAP, and Smac/Diablo (Cell Signaling Technology Inc.). After five washes in TBST, membranes were incubated in a 1:10,000 dilution of HRP-conjugated donkey antirabbit or mouse IgG (Amersham Pharmacia Biotech, Piscataway, NJ). The membrane was developed by ECL technique according to the manufacturer's protocol. Protein expression was quantitated from digitized autoradiography films using image analysis software (Adobe Systems, San Jose, CA). Protein levels were normalized by probing the same blot with GAPDH.

\section{Statistical analysis}

In groups with equal variances, datasets were analyzed using one-way ANOVA, and individual group means were then compared with the StudentNewman-Keuls multiple comparison test. In groups containing unequal variances, Kruskal-Wallis ANOVA was performed, followed by Dunnett's method for multiple comparisons. All values were expressed as the mean \pm SEM. Significance was assigned, where $P<0.05$. For percent change between groups, values obtained from negative controls were subtracted from each data point.

\section{RESULTS}

\section{Delayed neutrophil apoptosis in CLP-induced sepsis}

It is well known that neutrophils are programmed to undergo spontaneous apoptosis. It has been demonstrated that PI-3K and the ERK1/2 are involved in neutrophil resistance to apoptosis induced by inflammatory mediators such as C5a and GM-CSF $[13,14,19,20]$. To determine how these pathways may be involved in sepsis-induced delayed neutrophil apoptosis, we isolated neutrophils from control and CLP rats. These cells were cultured with $20 \mu \mathrm{M}$ U0126 (MEK1/2 inhibitor), $50 \mu \mathrm{M} \mathrm{LY294002} \mathrm{(PI-3K} \mathrm{inhibitor),} \mathrm{or}$ $50 \mathrm{nM}$ C5a for a $20-\mathrm{h}$ incubation at $37^{\circ} \mathrm{C}$ in PRMI medium supplemented with $10 \%$ FBS. As shown in Figure 1A, nearly $55 \%$ of control neutrophils were apoptotic after $20 \mathrm{~h}$ of incubation, and only $22 \%$ of the neutrophils isolated from CLP rats showed evidence of apoptosis. MEK1/2 and PI-3K inhibitors had no significant effects on neutrophil apoptosis when the cells were isolated from control animals (control neutrophils). However, CLP neutrophils almost completely regained their apoptotic ability in the presence of the PI-3K inhibitor, but no effect was seen after in vitro exposure to the MEK1/2 inhibitor. As would be expected, C5a at a final concentration of $50 \mathrm{nM}$ slightly but significantly reduced 
A

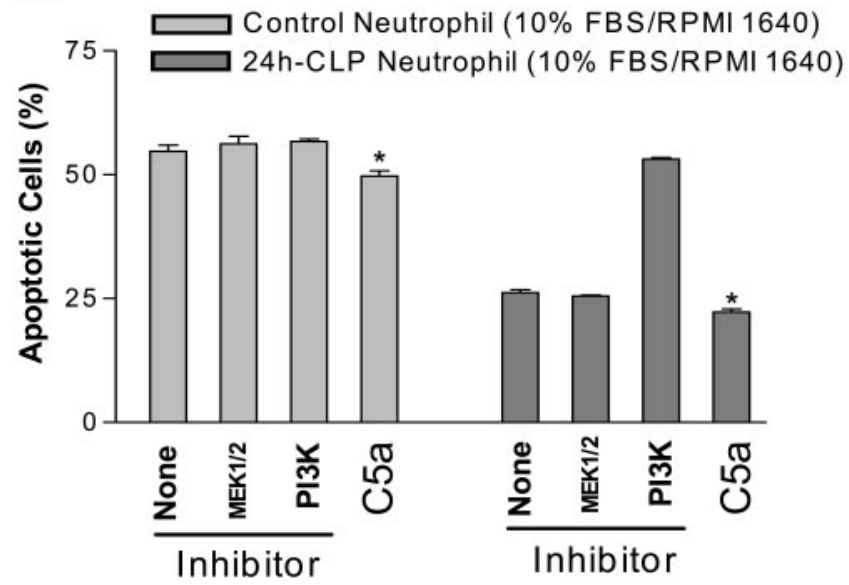

B

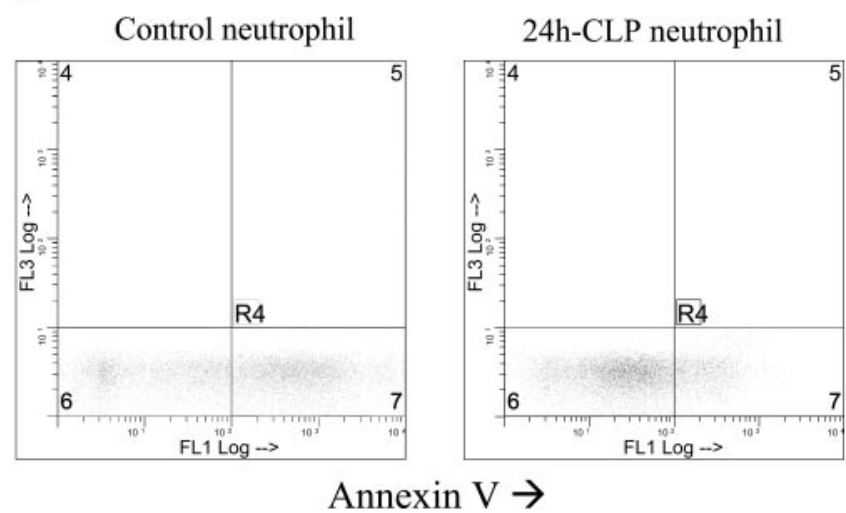

C Control Neutrophils

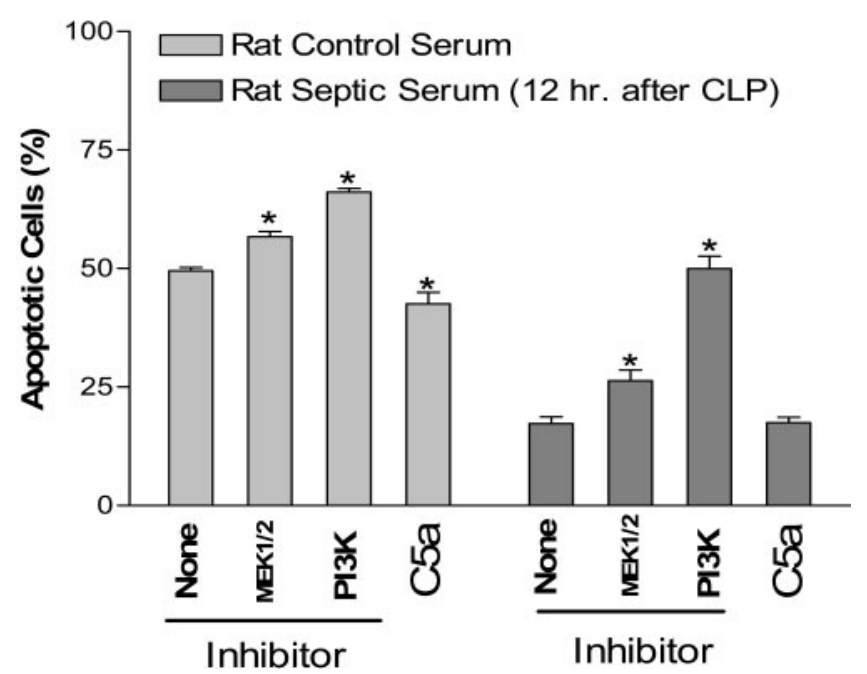

Fig. 1. Different apoptotic outcomes in neutrophils from septic and nonseptic rats. Blood neutrophils were isolated from normal (control) and CLP rats and incubated with $20 \mu \mathrm{M}$ U0126 (MEK1/2 inhibitor), $50 \mu \mathrm{M} \mathrm{LY} 294002$ (PI-3K inhibitor), or $50 \mathrm{nM}$ C5a for $20 \mathrm{~h}$ in PRMI medium supplemented with $10 \%$ FBS. After incubation, neutrophil apoptosis was assessed by flow cytometry for Ax-PI staining (A and B). Sera from rats were incubated with neutrophils (C). Neutrophils isolated from control animals were also incubated with $20 \mu \mathrm{M}$ U0126, $50 \mu \mathrm{M} \mathrm{LY294002,} \mathrm{or} 50 \mathrm{nM}$ C5a and cultured in PRMI medium supplemented with $10 \%$ rat control serum or $10 \%$ rat septic serum obtained $12 \mathrm{~h}$ after CLP. Neutrophil apoptosis was subsequently assessed after $20 \mathrm{~h}$ in vitro incubation at $37^{\circ} \mathrm{C}$. Data are representative of two to three independent experiments with neutrophils pooled from four to six rats. Samples were done in triplicate. *, $P<0.05$, compared with the "None/Inhibitor" group. neutrophil apoptosis. Dot-plot graphs (Ax vs. PI) were shown in Figure 1B to illustrate the different rate of apoptosis in control neutrophils and $24 \mathrm{~h}$ CLP neutrophils.

Sera from septic rats (12 h after CLP) were also tested for the antiapoptotic activity for neutrophils, which when isolated from control animals, were treated with $20 \mu \mathrm{M}$ U0126, $50 \mu \mathrm{M}$ LY294002, or $50 \mathrm{nM} \mathrm{C5a}$ and cultured in PRMI medium supplemented with $10 \%$ rat control serum or $10 \%$ rat septic serum. As shown in Figure 1C, the exposure of control neutrophils to rat septic serum strongly inhibited neutrophil apoptosis. Fifty percent of neutrophils underwent apoptosis when exposed to rat control serum, and only $18 \%$ of cells underwent apoptosis in the presence of rat septic serum. The decrease of neutrophil apoptosis could be partially attenuated by the MEK1/2 inhibitor and reversed completely by the PI-3K inhibitor (Fig. 1C, right panel). In contrast to the data obtained by using 10\% FBS (Fig. 1A, left panel), MEK1/2 and PI-3K inhibitors were able to cause increased neutrophil apoptosis in the presence of $10 \%$ rat control serum, suggesting that rat control serum may render more apoptotic signals to neutrophils sensitized by MEK1/2 or PI-3K inhibitor than commercial FBS. Exposure to C5a inhibited neutrophil apoptosis in the presence of rat control serum. However, delayed neutrophil apoptosis by septic serum could not be decreased further by exposure to C5a (Fig. 1C, right panel).

\section{Effects of septic sera on neutrophil apoptosis}

To further explore the mechanisms by which neutrophils are protected from apoptosis by septic sera, we evaluated the effects of septic sera on activation of MEK1/2 and PI-3K. Serum was isolated from rat blood $0,3,6,12$, and $24 \mathrm{~h}$ after CLP. As shown in Figure 2A, apoptosis of neutrophils from normal rats decreased dramatically when exposed to $10 \%$ septic serum from $3 \mathrm{~h}$ CLP rats, and further protection by sera was obtained 6, 12, and $24 \mathrm{~h}$ after CLP (Fig. 2A). Neutrophils were also treated with septic serum from $12 \mathrm{~h}$ CLP rats to evaluate its effects on the activation of PI-3K and MEK pathways. As shown in Figure 2B, septic serum, but not control serum, caused AKT phosphorylation as early as 5 min after exposure. The AKT activation was sustained to $30 \mathrm{~min}$ and disappeared by $1 \mathrm{~h}$ incubation. Septic serum was also able to cause marked phosphorylation of $\mathrm{p} 44 / 42$, and control serum had less effect on p44/42 activation (Fig. 2C). These data suggest that septic sera provide strong survival (antiapoptotic) signals for neutrophils by activation of MAPK and PI-3K pathways.

\section{Expression of $\mathrm{Bcl}-2$ family members in neutrophils during sepsis}

Blood neutrophils were isolated from control and CLP rats and then incubated with $10 \%$ control rat serum for $20 \mathrm{~h}$ at $37^{\circ} \mathrm{C}$. Before and after incubation, neutrophils were lysed in Laemmli buffer for Western blot analysis. As shown in Figure 3, Bcl-xL expression was up-regulated markedly in CLP neutrophils in comparison with control neutrophils (lane 1 vs. lane 3 ), and Bim expression diminished in CLP neutrophils (lane 1 vs. lane 3). BAD and BAX levels were not altered significantly. All these Bcl-2 members showed a rapid rate of protein degrada- 
A
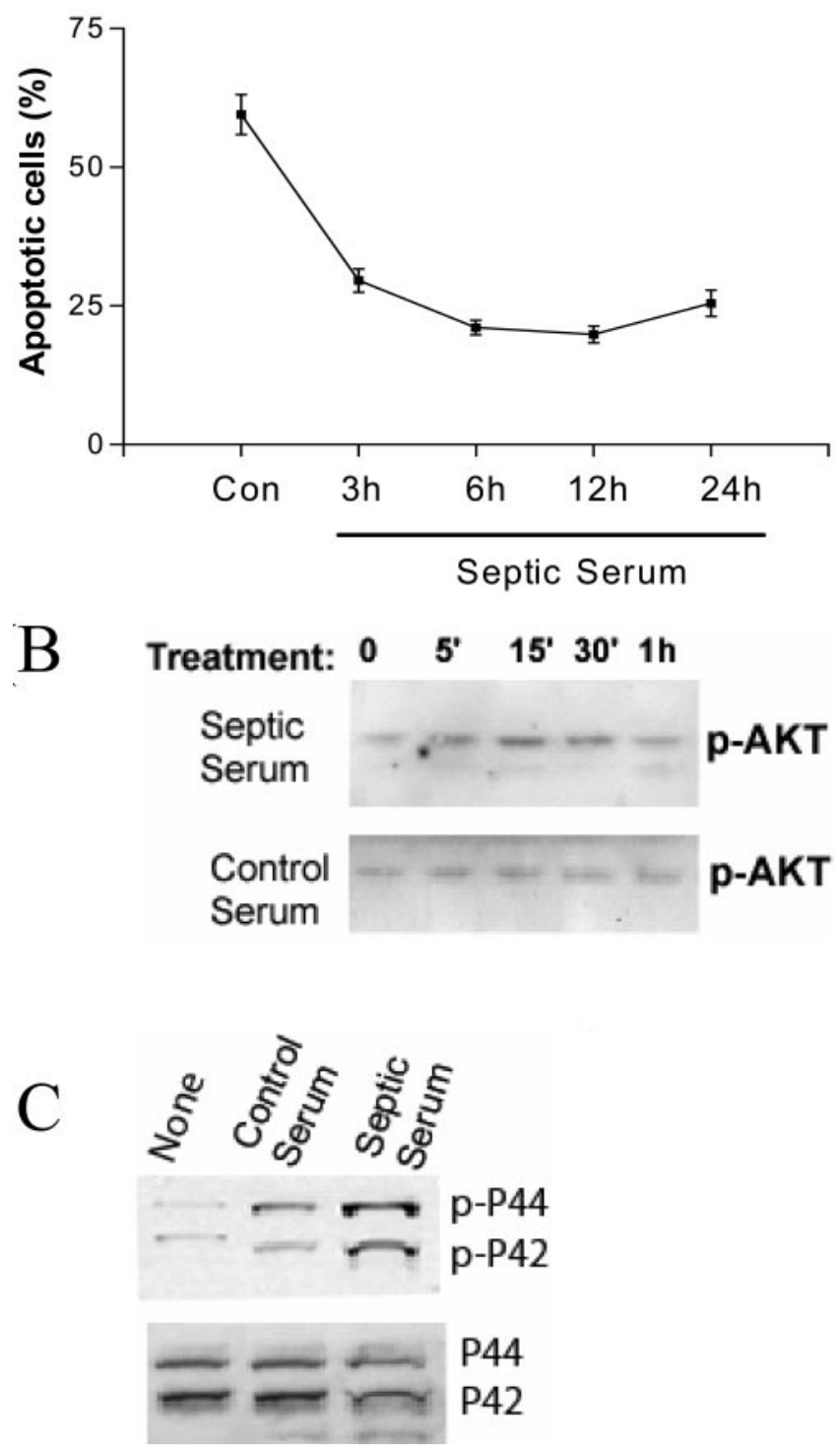

Fig. 2. Effects of septic sera on neutrophil apoptosis. Serum was isolated from rat blood 0, 3, 6, 12, and $24 \mathrm{~h}$ after CLP. Neutrophils from control rats were cultured with $10 \%$ septic serum for $20 \mathrm{~h}$, and neutrophil apoptosis was subsequently evaluated as described above (A). To explore the mechanism by which neutrophils are protected from apoptosis by septic sera $(12 \mathrm{~h}$ after CLP), we evaluated the effects of septic sera on phosphorylation of AKT and $\mathrm{p} 44 / 42$ (p-AKT and p-P44/42, respectively) by Western blot analysis (B and C). Approximately $5 \times 10^{6}$ cells per condition were used for whole cell lysis, using $100 \mu \mathrm{l}$ Laemmli buffer containing 5\% 2-ME. Data are representative of three independent experiments with neutrophils pooled from four to six rats.

tion in control and CLP neutrophils after $20 \mathrm{~h}$ in vitro incubation, suggesting that other intracellular proteins besides Bcl-2 members may be involved in delaying neutrophils from undergoing apoptosis during sepsis.

In vivo and in vitro role of $\mathrm{C} 5 \mathrm{a}$ in neutrophil apoptosis

Plasma samples were collected at $0,6,12,24,36$, and $48 \mathrm{~h}$ after CLP, and C5a levels in these plasma samples were determined by ELISA. The plasma C5a levels peaked at 12-24 $\mathrm{h}$, showing a 2.5 -fold increase over the 0 time samples, which contained $3.0 \mathrm{ng}$ C5a per milliliter. C5a levels declined thereafter. However, it is reasonable to postulate that C5a levels in septic plasma are much higher than those determined by ELISA, as C5a is known to bind quickly to C5aR on neutrophils and internalize.

To appraise the role of C5a in delayed neutrophil apoptosis in sepsis, $500 \mu \mathrm{g}$ anti-C5a $\mathrm{IgG}$ or preimmune (Control) $\mathrm{IgG}$ was administered i.v. into rats immediately after CLP. Neutrophils were obtained from rats $12 \mathrm{~h}$ after CLP and incubated in vitro for $20 \mathrm{~h}$ at $37^{\circ} \mathrm{C}$. Neutrophils were then assessed for apoptosis. Consistent with the findings described above, neutrophils from septic animals showed a low rate of apoptosis $(27 \%)$. However, anti-C5a treatment increased the neutrophil apoptosis rate significantly, to $40.6 \%(P<0.01$; Fig. 4), suggesting that endogenously generated C5a plays an important role in regulation of neutrophil apoptosis during sepsis.

C5a has been shown to regulate neutrophil expression of many inflammatory mediators in synergy with LPS [21-23]. To investigate if LPS and C5a also coregulate neutrophil apoptosis in vitro, neutrophils from control rats were incubated for $20 \mathrm{~h}$

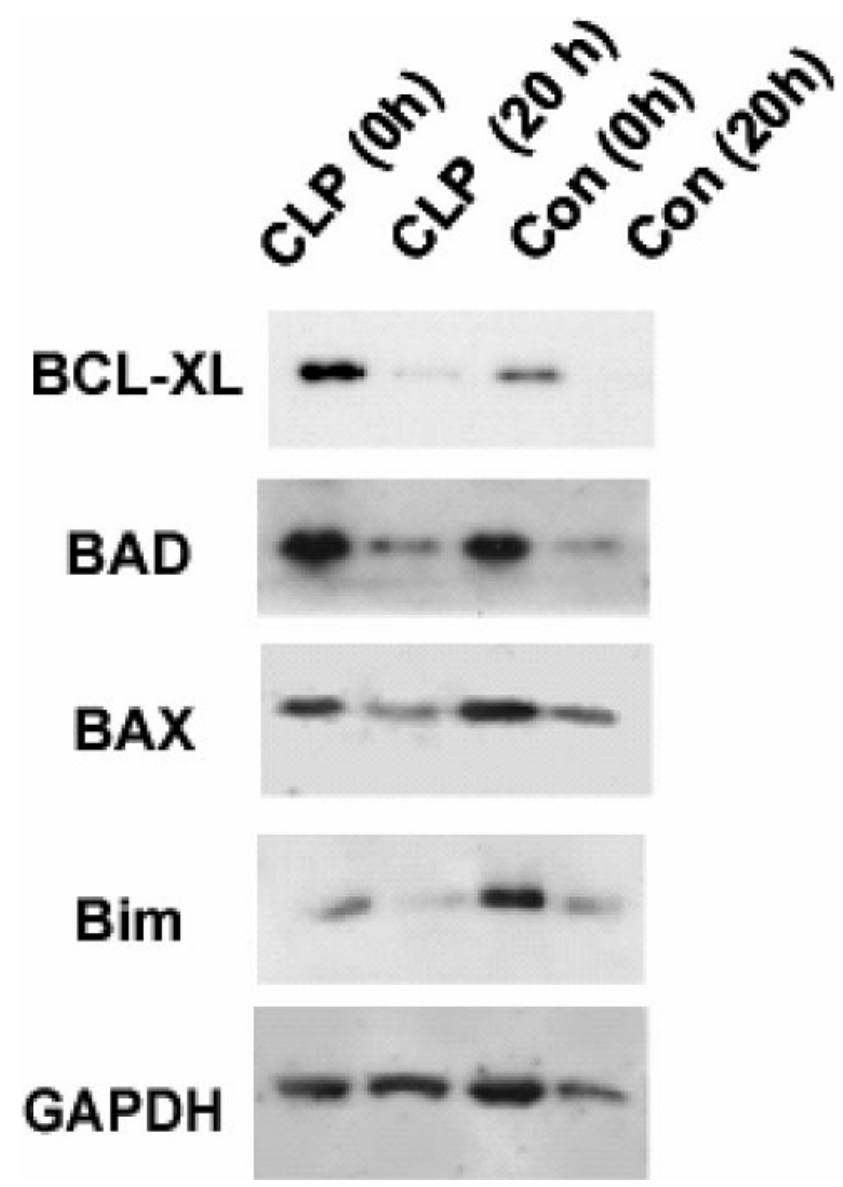

Fig. 3. Western blot analysis of expression of Bcl-2 family members in neutrophils during sepsis. Neutrophils were isolated from control rats (Con) and $20 \mathrm{~h}$ after CLP rats and incubated with $10 \%$ control rat serum for $20 \mathrm{~h}$. Before and after incubation, neutrophils were lysed immediately in Laemmli buffer for Western blot analysis. Data are representative of two to three independent experiments with neutrophils pooled from four to six rats. 


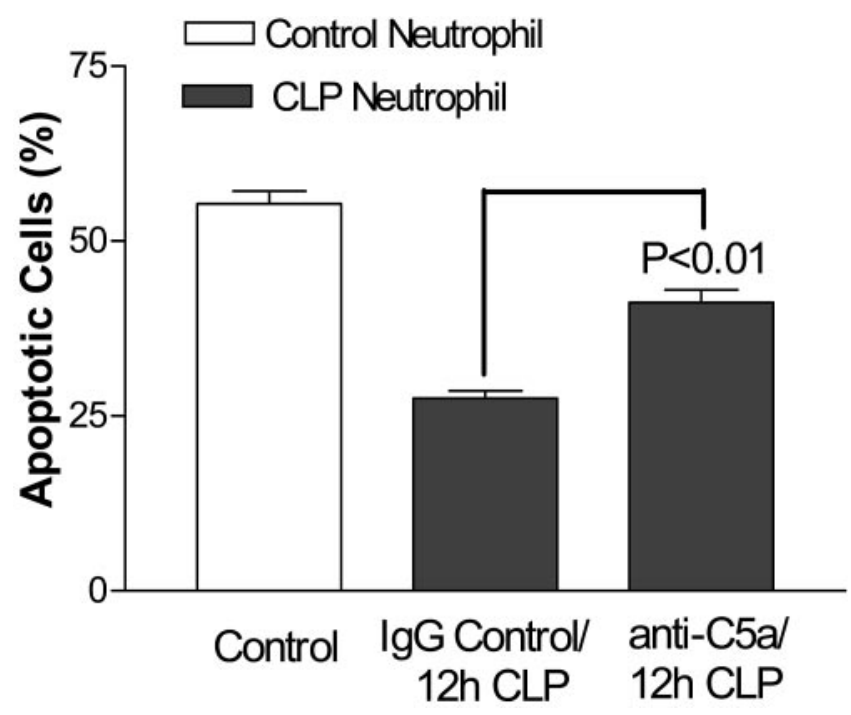

Fig. 4. Role of C5a in neutrophil apoptosis during sepsis in vivo. To assess the role of C5a in delayed neutrophil apoptosis in sepsis, $500 \mu \mathrm{g}$ anti-C5a IgG was administered i.v. to rats immediately after CLP. Neutrophils were obtained from rats $12 \mathrm{~h}$ after CLP and incubated at $37^{\circ} \mathrm{C}$ in vitro for $20 \mathrm{~h}$. Neutrophil apoptosis was then assessed to evaluate the role of anti-C5a treatment on neutrophil apoptosis in sepsis. Data are representative of three independent experiments. Neutrophils were pooled from four to six rats. For each vertical bar, $n=4$.

at $37^{\circ} \mathrm{C}$ in the presence of C5a, LPS, or the combination. It is interesting that C5a, LPS, and LPS + C5a had equal inhibitory effects on neutrophils apoptosis ( $\sim 15 \%$ reduction; Fig. 5A). No synergistic or additive effects were found with the combination of LPS and C5a. As shown in Figure 5, B and C, exposure to C5a (but not to LPS) resulted in AKT and p44/42 phosphorylation, suggesting specific agonist effects of C5a on AKT and MAPK signaling pathways.

To further explore the influence of C5a and LPS on proand antiapoptotic molecules, we evaluated the expression pattern of XIAP, Smac/Diablo, Bcl-xL, and BAX in response to stimulation with C5a or LPS or the combination. Neutrophils isolated from control rats were exposed to C5a (50 nM), LPS (100 ng), or LPS + C5a for $4 \mathrm{~h}$, and Western blot analysis was performed to assess the levels of these proteins. As shown in Figure 6, C5a and LPS were able to induce XIAP expression, and the combination of these inflammatory mediators failed to show additive effects on induction of XIAP expression. The cell protein levels of BAX and Smac/ Diablo were not obviously altered by C5a or LPS or the combination. C5a and LPS were capable of inducing Bcl-xL expression, but no additive effect was obvious when the combination of LPS and C5a was used. These data suggest that C5a and LPS may inhibit neutrophil apoptosis through up-regulating expression of antiapoptotic molecules (XIAP and Bcl-xL) and that C5a and LPS signaling may overlap on induction of antiapoptotic molecule expression. The effect of septic serum on the expression of these molecules was also shown in Figure 6. Septic serum showed a significant induction on XIAP and Bcl-xL expression but showed no effect on Smac/Diablo and BAX expression.

\section{DISCUSSION}

It is well known that sepsis triggers complement activation [10]. Excessive activation of complement leads to high levels of complement activation products in the blood, including the potent proinflammatory peptides, C3a and C5a. In humans and animals with sepsis, elevated plasma levels of C3a, C4a, and C5a have been described [24-27]. Excessive production of C5a during sepsis is detrimental. Indeed, C5a has been shown to play an essential role in the pathogenesis of sepsis [10]. In neutrophils, low (1-10 nM) doses of C5a can lead to nonspecific, chemotactic "desensitization," thereby causing broad dysfunction as well as paralysis of MAPK pathways. In sepsis, blood neutrophils from CLP rats display defective phagocytosis and chemotaxis as well as defective assembly of NADPH oxidase [28]. All such defects can be prevented by in vivo blockade of C5a or C5aR, suggesting that neutrophil innate immune functions in sepsis are seriously compromised by overproduction of C5a and excessive engagement of C5aR.

C5a/C5aR signaling on neutrophils appears to provide survival signals for these cells. High levels of neutrophil sequestration may result in tissue damage by releasing proteinases and free radicals, ultimately leading to multiple organ failure. In humans, spontaneous apoptosis was reduced significantly (only 8.6\%) in blood neutrophils obtained from patients with SIRS when compared with neutrophils from normal donors $(34.9 \%)$ [5]. Our in vitro experiments demonstrate that more than $50 \%$ of neutrophils isolated from normal donors undergo apoptosis during a 20 - $\mathrm{h}$ in vitro culture process (at $37^{\circ} \mathrm{C}$ ). Exposure of neutrophils to C5a can inhibit neutrophil apoptosis in a dose- and time-dependent manner. The antiapoptotic effects of C5a were decreased markedly in the presence of a wortmannin (a PI-3K) inhibitor or PD98059 (an ERK inhibitor), suggesting that the PI-3K/Akt and the MEK pathways are required for induction of apoptosis [13, 14]. In this study, we demonstrated that the PI-3K pathway was a major pathway involving suppression of neutrophil apoptosis during sepsis, given the fact that the PI-3K inhibitor completely abolished the protective effects of sepsis on neutrophil apoptosis (Fig. 1, A and B). The protective role of the MEK pathway in neutrophil apoptosis seems to be limited in sepsis, as inhibition of the MEK1/2 pathway did not affect neutrophil survival from septic animals but partially reversed the suppression of neutrophil apoptosis induced by septic sera (see Fig. 1). These data suggest that the PI-3K/Akt pathway is likely to serve as the upstream machinery providing the essential survival signaling to neutrophils, and the MEK pathway may only serve as a branch in the context of prevention of neutrophil apoptosis. Under septic condition, how these two pathways cross-talk with each other is unknown. As also shown in Figure 1, the inhibitors of MEK1/2 and PI-3K did not exert any effect on the rate of apoptosis when neutrophils were cultured in the presence of 10\% FBS but enhanced apoptosis significantly when cultured in the presence of rat control serum. It is possible that the inhibition of MEK1/2 or PI-3K renders the cells to a mode that is sensitive to apoptosis, and vital factors in rat control serum, which might not exist in the FBS, promote neutrophil apoptosis. It needs to be pointed out that FBS and rat serum were not 


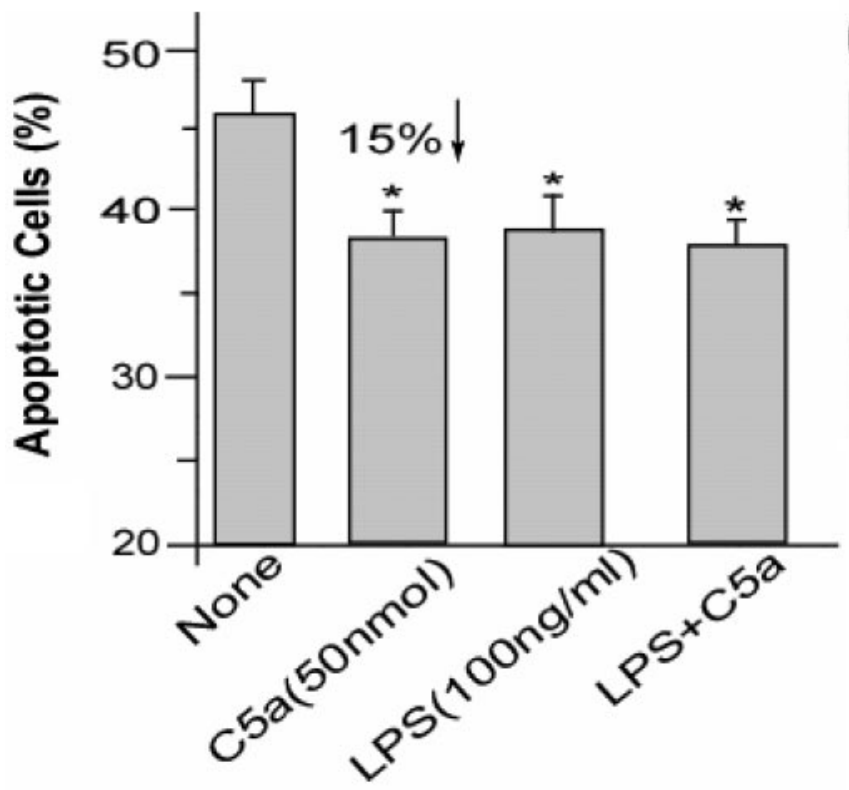

\section{Con C5a LPS LPS+C5a}

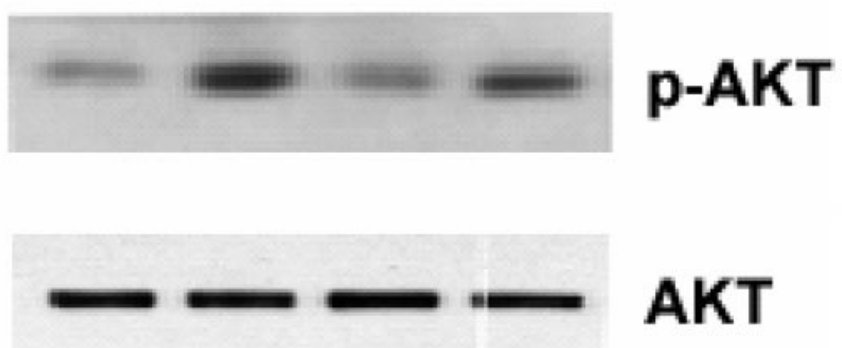

$\mathrm{C}$

Con C5a LPS LPS+C5a
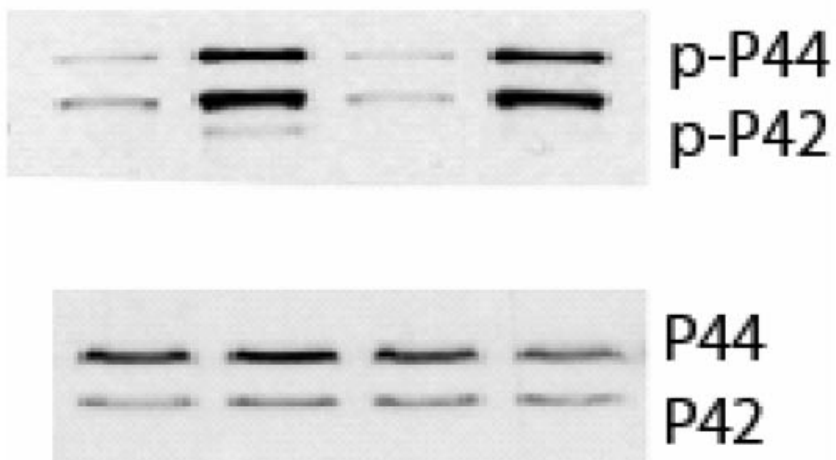

heat-inactivated to destroy the vital factors that are important for neutrophil apoptosis.

Neutrophil exposure to C5a leads to phosphorylation of Akt and Bad proteins in neutrophils as well as decreased activity of caspase-9 [14], suggesting that neutrophils undergo spontaneous apoptosis via a mitochondria-dependent pathway and that this pathway can be inhibited by C5a/C5aR signaling. As would be expected, blood neutrophils isolated from CLP rats were more resistant to apoptotic induction after $20 \mathrm{~h}$ of in vitro incubation when compared with blood neutrophils from normal rats. When CLP rats were treated with anti-C5a, blood neutrophils markedly regained the ability to undergo apoptosis in vitro (Fig. 4), suggesting that the long lifespan of neutrophils in sepsis is attributable, at least in part, to C5a generated during sepsis. The effects of anti-C5a treatment in vivo in restoring susceptibility to neutrophil apoptosis in vitro were more impressive than the in vitro exposure of neutrophils to C5a, which
Fig. 5. Role of C5a in neutrophil apoptosis in vitro. To investigate the effects of C.5a and LPS on neutrophil apoptosis, neutrophils from control rats were incubated for $20 \mathrm{~h}$ at $37^{\circ} \mathrm{C}$ in the presence of $\mathrm{C} 5 \mathrm{a}(50 \mathrm{nM})$, LPS $(100 \mathrm{ng} / \mathrm{ml})$, or LPS + C5a. Neutrophil apoptosis was subsequently assessed (A). The effects of C5a and LPS on phosphorylation of AKT and p42/44 were analyzed by Western blot (B and C). Approximately $5 \times 10^{6}$ cells were treated with C5a, LPS, or LPS + C5a for 5 min, and the cells were pelleted and lysed in $100 \mu \mathrm{l}$ Laemmli buffer containing 5\% 2-ME for Western blot analysis. Data are representative of two to three independent experiments with neutrophils pooled from four to six rats. only gave rise to a $15 \%$ protection in neutrophil apoptosis (Fig. 5), suggesting that C5a not only provides survival signals by itself but may also affect the expression of other inflammatory mediators, which are involved in neutrophil apoptosis. As mentioned earlier, many other inflammatory mediators have antiapoptotic effects on neutrophils, including G-CSF, GMCSF, fMLP, ATP, LTB4, IL-1 $\beta$, IL-2, IL-3, IL-6, IL-8, IL-15, and IFN- $\gamma$ [29]. It is well known that C5a/C5aR signaling influences mediators of the inflammatory network. C5a stimulated the synthesis and release from human neutrophils of proinflammatory cytokines such as TNF- $\alpha$, IL- $\beta$, IL-6, and IL-8 [30, 31]. Addition of low nanometer concentrations of C5a to HUVEC caused strong up-regulation of IL-8 and IL-1 $\beta$ mRNA in a time- and dose-dependent manner [32]. Blockade of C5a with anti-C5a in sepsis resulted in a $>75 \%$ decrease in serum levels of IL-6 [31]. In CLP-induced sepsis, we have demonstrated that blockade of $\mathrm{C} 5 \mathrm{aR}$ with antibodies also re- 

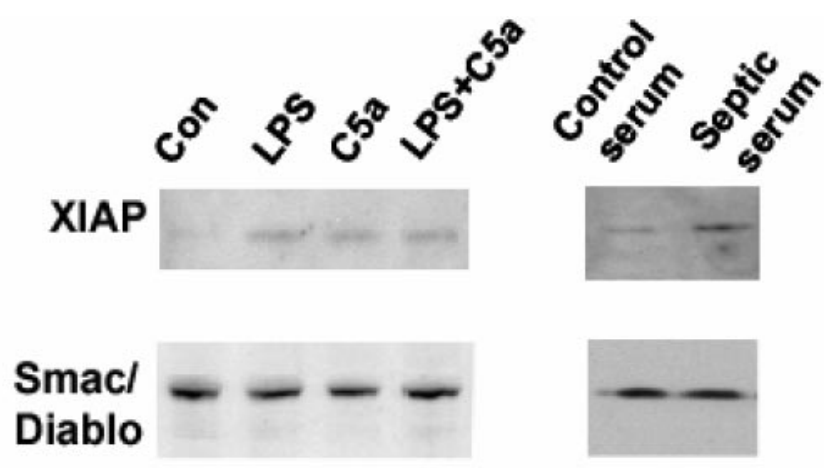

BCL-XL

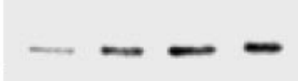

BAX
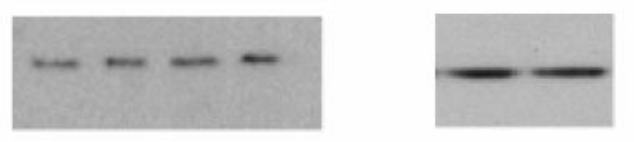

GAPDH


Fig. 6. Influence of C5a, LPS, and septic serum on pro- and antiapoptotic molecules. The expression patterns of XIAP, Smac/Diablo, Bcl-xL, and BAX were evaluated by Western blots after stimulation. Neutrophils isolated from control rats were treated with C5a (50 nM), LPS (100 ng/ml), the combination, or the septic serum $(10 \%)$ for $4 \mathrm{~h}$, and Western blot analysis was performed to assess the levels of these proteins. Data are representative of three independent experiments with neutrophils pooled from four to six rats.

sulted in decreased levels of IL-6 in blood compared with CLP controls [11]. The dependency of IL-6 production on C5a during sepsis was confirmed in C5aR-deficient mice, as IL-6 levels were significantly lower in comparison with control mice during sepsis. More recently, it has been demonstrated that macrophage migration inhibitory factor (MIF) is a survival cytokine for neutrophils [33] and that C5a is a positive regulator of MIF production during sepsis [18]. Conversely, in vivo blockade of MIF airway during sepsis in mice is protective [34]. These data suggest that C5a plays a pivotal role in the regulation of inflammatory mediator expression and may provide survival signals indirectly for neutrophils by increasing the concentrations of antiapoptotic inflammatory mediators in circulation during sepsis. The fundamental question here is whether apoptotic-resistant neutrophils are beneficial or harmful in the setting of sepsis.

In the current study, we observed that septic sera strongly inhibited neutrophil apoptosis, indicating extracellular signals for neutrophil survival mainly derived from unknown product(s) in septic serum. These sera were capable of activating PI-3K/AKT and MEK pathways, which are essential for neutrophil survival. It is interesting that the serum from an early time-point of $3 \mathrm{~h}$ CLP showed a high level of protection against neutrophil apoptosis, implying the presence of inflammatory mediators with antiapoptotic effects and appearing early after induction of sepsis. Thus, resistance of neutrophils to apoptosis is likely to be an early event, occurring in the early stages of sepsis.

Intracellular events that lead to neutrophil apoptosis have been studied extensively. Neutrophil apoptosis is mediated by caspases 3, 8, and 9 [2]. The rate of neutrophil apoptosis can be accelerated under high levels of oxidative stress. It has been shown that death receptor clustering and subsequent activation of caspase 8 are redox-sensitive and that high levels of oxidative stress may cause genotoxic injury by triggering the P53 pathway as well as DNA damage, thereby leading to apoptosis [35]. Involvement of caspase 9 suggests the role of mitochondria in neutrophil apoptosis. Occurrence of mitochondrial membrane depolarization in neutrophil apoptosis precedes activation of caspases and cell membrane expression of phosphatidylserine [36]. Moreover, Bcl-2 family members appear to play an important role in neutrophil survival. The antiapoptotic molecules, BcL-2 and Bcl-xL, act in many cell types to maintain the mitochondrial integrity by preventing loss of mitochondrial membrane potential and release of proapoptotic proteins such as cytochrome $\mathrm{C}$ into the cytosol, and the proapoptotic proteins, Bax, Bak, Bad, and Bim, promote release of cytochrome C, which leads to apoptosis. XIAP functions as an antiapoptotic molecule on a different level, by blocking the activation of caspases responsible for downstream execution of the apoptotic cascade. The antiapoptotic action of XIAP can be inhibited by its binding of Smac or Diablo. The Bcl-xL level in canine neutrophils was strongly up-regulated by LPS, being associated with resistance to apoptosis [37]. In vitro experiments demonstrated that Bcl-xL expression in neutrophils was up-regulated by C5a and LPS (Fig. 6). It is interesting that LPS and C5a were able to induce XIAP expression, but the additive effect was not seen with costimulation of neutrophils. Similarly, C5a and LPS did not show any synergistic or additive effect on neutrophil apoptosis in vitro (Fig. 5), suggesting that C5a and LPS signalings may overlap in the protection of neutrophil apoptosis. As would be expected, septic serum can also induce $\mathrm{XIAP}$ and Bcl-xL (Fig. 6). The XIAP gene is also known to be up-regulated in septic neutrophils [38, 39]. The XIAP/Smac/ Diablo pathway could be another mechanism that regulates neutrophil survival in sepsis. Thus, PI-3K and MEK pathways, as well as the XIAP/Smac/Diablo pathway and Bcl-2 family members may function coordinately to promote neutrophil survival (Fig. 7).

In summary, the data in this study support the concept that the survival signaling for neutrophils during sepsis derives from the inflammatory milieu, with C5a playing an essential role in neutrophil resistance to apoptosis during sepsis. The PI-3K/AKT but not the ERK pathway appears to play a major role in conferring resistance to apoptosis in neutrophils. The intracellular molecules Bcl-xL, Bim, and XIAP may participate in the antiapoptotic outcome in neutrophils during sepsis. In addition, anti-C5a treatment may prevent neutrophil accumulation in organs by causing a normal lifespan in neutrophils. Thus, it is likely that an improved understanding of apoptosis regulation will lead to new insights into sepsis development and may also provide important knowledge for the development of novel therapeutic strategies. 


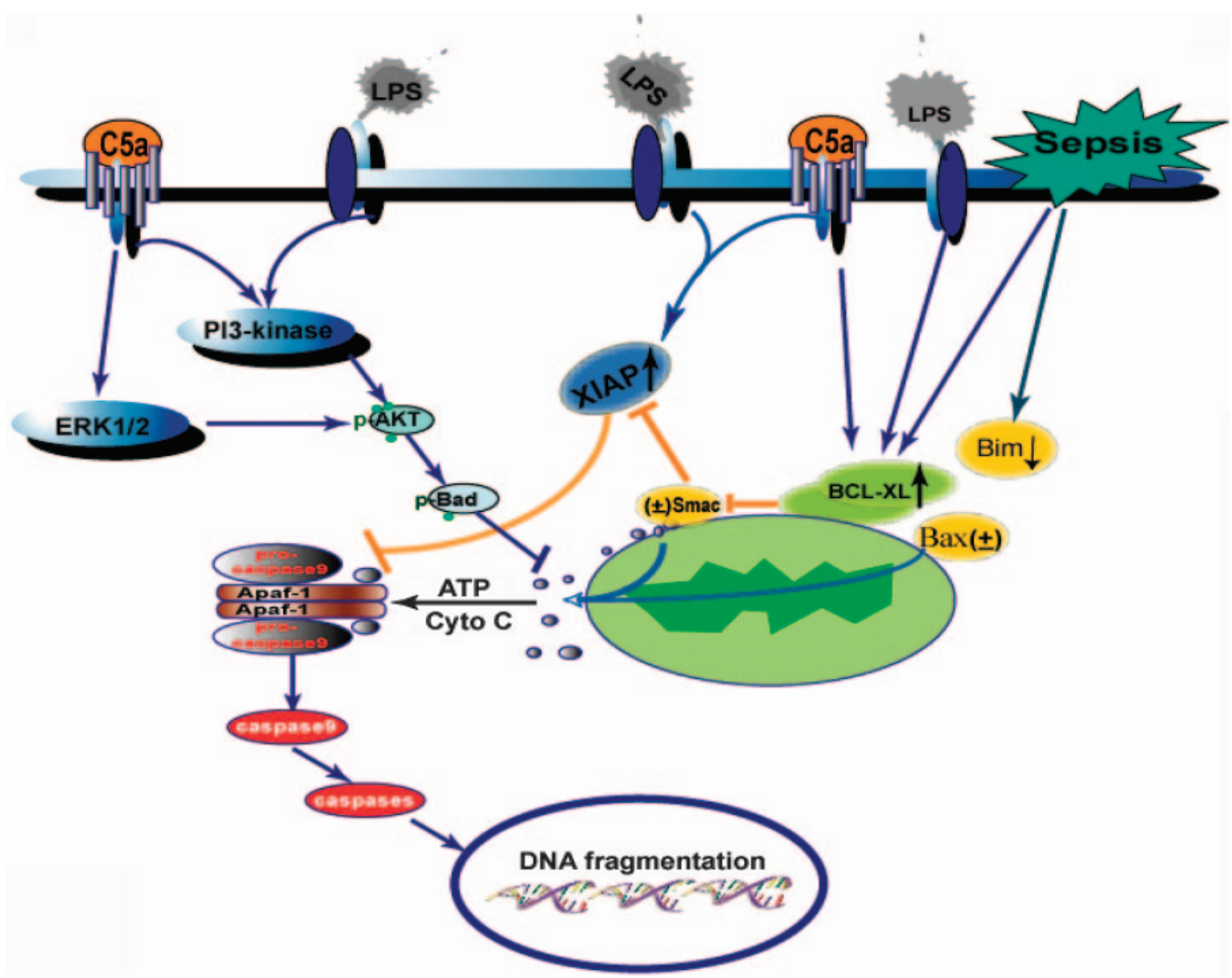

Fig. 7. Survival pathways of neutrophils in sepsis. C5a and LPS are presumed to play roles in sepsis. C5a activates ERK1/2 and PI-3K pathways, leading to phosphorylation of AKT and subsequent phosphorylation of Bad. Phosphorylated Bad inhibits cytochrome C release from mitochondria to prevent the formation of the apoptosome, thereby inhibiting neutrophil apoptosis. LPS and C5a can also induce XIAP production, which can inhibit the formation of the apoptosome. Sepsis enhances Bcl-xL expression and reduces Bim expression. C5a and LPS can also enhance Bcl-xL expression. All of these events are in favor of maintaining the integrity of mitochondria and preventing neutrophil apoptosis. Apaf-1, Apoptosis protease-activating factor.

\section{ACKNOWLEDGMENTS}

This work is supported by the National Institutes of Health (Grants GM-61656 and HL-31963). We thank Beverly Schumann for her secretarial assistance.

\section{REFERENCES}

1. Guo, R. F., Ward, P. A. (2002) Mediators and regulation of neutrophil accumulation in inflammatory responses in lung: insights from the $\operatorname{IgG}$ immune complex model. Free Radic. Biol. Med. 33, 303-310.

2. Webb, P. R., Wang, K. Q., Scheel-Toellner, D., Pongracz, J., Salmon, M., Lord, J. M. (2000) Regulation of neutrophil apoptosis: a role for protein kinase C and phosphatidylinositol-3-kinase. Apoptosis 5, 451-458.

3. Savill, J. S., Wyllie, A. H., Henson, J. E., Walport, M. J., Henson, P. M., Haslett, C. (1989) Macrophage phagocytosis of aging neutrophils in inflammation. Programmed cell death in the neutrophil leads to its recognition by macrophages. J. Clin. Invest. 83, 865-875.

4. Parsey, M. V., Kaneko, D., Shenkar, R., Abraham, E. (1999) Neutrophil apoptosis in the lung after hemorrhage or endotoxemia: apoptosis and migration are independent of interleukin-1 $\beta$. Chest 116, 67S-68S.

5. Jimenez, M. F., Watson, R. W., Parodo, J., Evans, D., Foster, D., Steinberg, M., Rotstein, O. D., Marshall, J. C. (1997) Dysregulated expression of neutrophil apoptosis in the systemic inflammatory response syndrome. Arch. Surg. 132, 1263-1269.
6. Mayadas, T. N., Cullere, X. (2005) Neutrophil $\beta 2$ integrins: moderators of life or death decisions. Trends Immunol. 26, 388-395.

7. Mecklenburgh, K., Murray, J., Brazil, T., Ward, C., Rossi, A. G., Chilvers, E. R. (1999) Role of neutrophil apoptosis in the resolution of pulmonary inflammation. Monaldi Arch. Chest Dis. 54, 345-349.

8. Wickel, D. J., Cheadle, W. G., Mercer-Jones, M. A., Garrison, R. N (1997) Poor outcome from peritonitis is caused by disease acuity and organ failure, not recurrent peritoneal infection. Ann. Surg. 225, 744753.

9. Walley, K. R., Lukacs, N. W., Standiford, T. J., Strieter, R. M., Kunkel, S. L. (1997) Elevated levels of macrophage inflammatory protein 2 in severe murine peritonitis increase neutrophil recruitment and mortality. Infect. Immun. 65, 3847-3851.

10. Guo, R. F., Ward, P. A. (2005) Role of C5a in inflammatory responses. Annu. Rev. Immunol. 23, 821-852.

11. Riedemann, N. C., Guo, R. F., Neff, T. A., Laudes, I. J., Keller, K. A., Sarma, V. J., Markiewski, M. M., Mastellos, D., Strey, C. W., Pierson, C. L., Lambris, J. D., Zetoune, F. S., Ward, P. A. (2002) Increased C5a receptor expression in sepsis. J. Clin. Invest. 110, 101-108.

12. Guo, R. F., Riedemann, N. C., Bernacki, K. D., Sarma, V. J., Laudes, I. J., Reuben, J. S., Younkin, E. M., Neff, T. A., Paulauskis, J. D., Zetoune, F. S., Ward, P. A. (2003) Neutrophil C5a receptor and the outcome in a rat model of sepsis. FASEB J. 17, 1889-1891.

13. Perianayagam, M. C., Balakrishnan, V. S., King, A. J., Pereira, B. J., Jaber, B. L. (2002) C5a delays apoptosis of human neutrophils by a phosphatidylinositol 3-kinase-signaling pathway. Kidney Int. 61, 456463.

14. Perianayagam, M. C., Balakrishnan, V. S., Pereira, B. J., Jaber, B. L. (2004) C5a delays apoptosis of human neutrophils via an extracellular 
signal-regulated kinase and Bad-mediated signaling pathway. Eur. J. Clin. Invest. 34, 50-56.

15. Guo, R. F., Huber-Lang, M., Wang, X., Sarma, V., Padgaonkar, V. A., Craig, R. A., Riedemann, N. C., McClintock, S. D., Hlaing, T., Shi, M. M., Ward, P. A. (2000) Protective effects of anti-C5a in sepsis-induced thymocyte apoptosis. J. Clin. Invest. 106, 1271-1280.

16. Huber-Lang, M. S., Sarma, J. V., McGuire, S. R., Lu, K. T., Guo, R. F., Padgaonkar, V. A., Younkin, E. M., Laudes, I. J., Riedemann, N. C., Younger, J. G., Ward, P. A. (2001) Protective effects of anti-C5a peptide antibodies in experimental sepsis. FASEB J. 15, 568-570.

17. Riedemann, N. C., Guo, R. F., Hollmann, T. J., Gao, H., Neff, T. A., Reuben, J. S., Speyer, C. L., Sarma, J. V., Wetsel, R. A., Zetoune, F. S., Ward, P. A. (2004) Regulatory role of C5a in LPS-induced IL-6 production by neutrophils during sepsis. FASEB J. 18, 370-372.

18. Riedemann, N. C., Guo, R. F., Gao, H., Sun, L., Hoesel, M., Hollmann, T. J., Wetsel, R. A., Zetoune, F. S., Ward, P. A. (2004) Regulatory role of C5a on macrophage migration inhibitory factor release from neutrophils. J. Immunol. 173, 1355-1359.

19. Klein, J. B., Rane, M. J., Scherzer, J. A., Coxon, P. Y., Kettritz, R., Mathiesen, J. M., Buridi, A., McLeish, K. R. (2000) Granulocyte-macrophage colony-stimulating factor delays neutrophil constitutive apoptosis through phosphoinositide 3-kinase and extracellular signal-regulated kinase pathways. J. Immunol. 164, 4286-4291.

20. Kotone-Miyahara, Y., Yamashita, K., Lee, K. K., Yonehara, S., Uchiyama, T., Sasada, M., Takahashi, A. (2004) Short-term delay of Fas-stimulated apoptosis by GM-CSF as a result of temporary suppression of FADD recruitment in neutrophils: evidence implicating phosphatidylinositol 3-kinase and MEK1-ERK1/2 pathways downstream of classical protein kinase C. J. Leukoc. Biol. 76, 1047-1056.

21. Riedemann, N. C., Guo, R. F., Sarma, V. J., Laudes, I. J., Huber-Lang, M., Warner, R. L., Albrecht, E. A., Speyer, C. L., Ward, P. A. (2002) Expression and function of the C5a receptor in rat alveolar epithelial cells. J. Immunol. 168, 1919-1925.

22. Laudes, I. J., Chu, J. C., Huber-Lang, M., Guo, R. F., Riedemann, N. C., Sarma, J. V., Mahdi, F., Murphy, H. S., Speyer, C., Lu, K. T., Lambris, J. D., Zetoune, F. S., Ward, P. A. (2002) Expression and function of C5a receptor in mouse microvascular endothelial cells. J. Immunol. 169, $5962-5970$

23. Riedemann, N. C., Guo, R. F., Bernacki, K. D., Reuben, J. S., Laudes, I. J., Neff, T. A., Gao, H., Speyer, C., Sarma, V. J., Zetoune, F. S., Ward, P. A. (2003) Regulation by C5a of neutrophil activation during sepsis. Immunity 19, 193-202.

24. de Boer, J. P., Creasey, A. A., Chang, A., Roem, D., Eerenberg, A. J., Hack, C. E., Taylor Jr., F. B. (1993) Activation of the complement system in baboons challenged with live Escherichia coli: correlation with mortality and evidence for a biphasic activation pattern. Infect. Immun. 61, 42934301.

25. Smedegard, G., Cui, L. X., Hugli, T. E. (1989) Endotoxin-induced shock in the rat. A role for C5a. Am. J. Pathol. 135, 489-497.
26. Bengtson, A., Heideman, M. (1988) Anaphylatoxin formation in sepsis. Arch. Surg. 123, 645-649.

27. Nakae, H., Endo, S., Inada, K., Takakuwa, T., Kasai, T., Yoshida, M. (1994) Serum complement levels and severity of sepsis. Res. Commun. Chem. Pathol. Pharmacol. 84, 189-195.

28. Huber-Lang, M. S., Younkin, E. M., Sarma, J. V., McGuire, S. R., Lu, K. T., Guo, R. F., Padgaonkar, V. A., Curnutte, J. T., Erickson, R., Ward, P. A. (2002) Complement-induced impairment of innate immunity during sepsis. J. Immunol. 169, 3223-3231.

29. Simon, H. U. (2003) Neutrophil apoptosis pathways and their modifications in inflammation. Immunol. Rev. 193, 101-110.

30. Strieter, R. M., Kasahara, K., Allen, R. M., Standiford, T. J., Rolfe, M. W., Becker, F. S., Chensue, S. W., Kunkel, S. L. (1992) Cytokine-induced neutrophil-derived interleukin-8. Am. J. Pathol. 141, 397-407.

31. Hopken, U., Mohr, M., Struber, A., Montz, H., Burchardi, H., Gotze, O., Oppermann, M. (1996) Inhibition of interleukin-6 synthesis in an animal model of septic shock by anti-C5a monoclonal antibodies. Eur. J. Immunol. 26, 1103-1109.

32. Monsinjon, T., Gasque, P., Chan, P., Ischenko, A., Brady, J. J., Fontaine, M. C. (2003) Regulation by complement C3a and C5a anaphylatoxins of cytokine production in human umbilical vein endothelial cells. FASEB J. 17, 1003-1014

33. Baumann, R., Casaulta, C., Simon, D., Conus, S., Yousefi, S., Simon, H. U. (2003) Macrophage migration inhibitory factor delays apoptosis in neutrophils by inhibiting the mitochondria-dependent death pathway. FASEB J. 17, 2221-2230.

34. Calandra, T., Echtenacher, B., Roy, D. L., Pugin, J., Metz, C. N., Hultner, L., Heumann, D., Mannel, D., Bucala, R., Glauser, M. P. (2000) Protection from septic shock by neutralization of macrophage migration inhibitory factor. Nat. Med. 6, 164-170.

35. Melley, D. D., Evans, T. W., Quinlan, G. J. (2005) Redox regulation of neutrophil apoptosis and the systemic inflammatory response syndrome. Clin. Sci. (Lond.) 108, 413-424.

36. Fossati, G., Moulding, D. A., Spiller, D. G., Moots, R. J., White, M. R., Edwards, S. W. (2003) The mitochondrial network of human neutrophils: role in chemotaxis, phagocytosis, respiratory burst activation, and commitment to apoptosis. J. Immunol. 170, 1964-1972.

37. Sano, J., Oguma, K., Kano, R., Yazawa, M., Tsujimoto, H., Hasegawa, A. (2005) High expression of Bcl-xL in delayed apoptosis of canine neutrophils induced by lipopolysaccharide. Res. Vet. Sci. 78, 183-187.

38. Hu, Z., Sayeed, M. M. (2004) Suppression of mitochondria-dependent neutrophil apoptosis with thermal injury. Am. J. Physiol. Cell Physiol. 286, C170-C178.

39. O'Neill, A. J., Doyle, B. T., Molloy, E., Watson, C., Phelan, D., Greenan, M. C., Fitzpatrick, J. M., Watson, R. W. (2004) Gene expression profile of inflammatory neutrophils: alterations in the inhibitors of apoptosis proteins during spontaneous and delayed apoptosis. Shock 21, 512-518. 